\title{
Evaluation of Novel Adhesive Film Containing Ketorolac for Post-Surgery Pain Control: A Safety and Efficacy Study
}

Khalid Al-Hezaimi, ${ }^{*}$ Mansour Al-Askar, ${ }^{*}$ Zuied Selamhe, ${ }^{\dagger}$ Jia-Hui Fu, ${ }^{\ddagger}$ Ibrahim A. Alsarra,, and Hom-Lay Wang*‡

Background: Prescribing analgesics after periodontal surgery is a common practice. However, it can become a challenge for patients with systemic diseases or who are on long-term medications. Ketorolac tromethamine (KT), a nonsteroidal anti-inflammatory drug, is incorporated into an adhesive film to overcome the limitations associated with oral, intravenous, intramuscular, or sublingual routes of drug administration. This study evaluates the analgesic effect of a KT adhesive film for pain management after periodontal surgery.

Methods: Aqueous solvents of two bioadhesive polymers (hydroxypropyl methylcellulose and polyacrylic acid), together with $30 \mathrm{mg}$ of KT, were used to formulate the adhesive film. Sixty-eight patients, who each received a free gingival graft, were randomly divided into treatment and control groups. In the treatment group, the prepared adhesive film was applied over the surgical site, whereas in the control group adhesive film without KT was placed initially. Two hours after surgery, the KT adhesive film was applied on the surgical site in the control group. A visual analog scale was used to assess the degree of pain encountered at $0,1,2,3,4,5,24$, and 48 hours post-surgery.

Results: The treatment group reported a significant reduction of pain intensity during the first 2 hours after surgery ( $P$ $<0.05)$. After the KT adhesive film was applied in the control group, pain intensity was reduced to a non-significant level by the third hour after surgery. No adverse reaction or undesirable gastrointestinal side effect was observed.

Conclusion: Adhesive film containing $30 \mathrm{mg}$ of KT was effective in controlling post-surgical pain with no observable gastrointestinal effects. J Periodontol 2011;82:963-968.

\section{KEY WORDS}

Adhesives; anti-inflammatory agents, non-steroidal; ketorolac; pain; periodontal diseases; surgery.

\footnotetext{
* Eng. A.B. Research Chair for Growth Factors and Bone Regeneration, Division of Periodontics, College of Dentistry, King Saud University, Riyadh, Saudi Arabia.

$\dagger$ Eng. A.B. Research Chair for Growth Factors and Bone Regeneration, College of Dentistry, King Saud University.

$\ddagger$ Department of Periodontics and Oral Medicine, School of Dentistry, University of Michigan, Ann Arbor, MI

$\S$ Department of Pharmaceutics, College of Pharmacy, Center of Excellence in Biotechnology Research, King Saud University.
}

I $\mathrm{t}$ was observed that significant postoperative pain has been associated with free soft tissue grafts or subepithelial connective tissue grafts. ${ }^{1-3}$ Hence, pain management after performing these procedures is of paramount importance. In general, a prescription of oral analgesics is the most common method to manage post-surgical pain. Given the vast array of analgesics available, the multiple medical conditions of the patients, and possible drug interactions or allergies, the clinician is often challenged when choosing an effective and safe analgesic for use after periodontal surgery. ${ }^{4}$

Ketorolac tromethamine (KT), a non-steroidal anti-inflammatory agent (NSAID), is currently administered intramuscularly and orally in multiple divided doses for short-term management of postoperative pain. ${ }^{5,6}$ Intramuscular injection is the preferred route of administration (30 mg, four times a day). It is administered for moderate-to-severe pain management, even though patient compliance is rather low for this route. ${ }^{7}$ Therefore, oral administration of KT (10 $\mathrm{mg}$, one tablet every 8 hours) has been used in the management of mild to moderate pain. ${ }^{8}$ Unfortunately, because KT is a non-specific inhibitor of the cyclooxygenase (COX) isozymes 1 and 2, it is associated with COX-1-mediated adverse 
effects, such as gastrointestinal (GI) erosions, ulcers and bleeding, inhibition of platelet function, and renal failure. ${ }^{9,10}$ Elderly individuals, patients with a history of GI complications, patients on multiple NSAID therapy, smokers, alcoholics, and individuals who have the Helicobacter pylori microorganism are most susceptible. ${ }^{9}$

Several research attempts have been conducted to formulate KT into dosage forms other than intramuscular injection or oral tablet. The feasibility of transdermal delivery of ketorolac as transdermal patches has been reported. ${ }^{11}$ Despite the immense advantages of this route, the limited percutaneous administration because of low skin permeability is the rate-limiting step of transdermal delivery of KT. ${ }^{12}$ To overcome this problem, a number of formulation strategies have been developed to enhance dermal delivery of KT, such as the use of penetration enhancers, ultrasound, and prodrug approach. However, these sophisticated techniques may alter the thermodynamic activity. ${ }^{13,14}$ Studies using nasal formulation of ketorolac have also been reported. ${ }^{15,16}$ However, the discomfort and inconvenience of this route has limited its use. Topical anti-inflammatory activity of ketorolac has exhibited acceptable efficiency for external use; however, penetration enhancers may be unavoidable in dermatologic formulations. ${ }^{17}$ Several studies have demonstrated that KT $0.4 \%$ ophthalmic solution, a recent reformulation of the original KT $0.5 \%$ solution, is used as an adjunct to steroids for the reduction of ocular pain and burning and stinging after cataract and refractive surgery. ${ }^{18}$ In the management of periodontal diseases, KT has been used as an oral rinse with a concentration of $0.1 \%$ and favorable treatment outcomes, such as prevention of alveolar bone loss, has been reported. ${ }^{19}$

Thus, developing an alternative dosage form that is easy to administer, is painless, is non-invasive, is easy to comply with, and avoids first-pass metabolism is worthwhile. Because KT has a short half-life (4 to 6 hours), frequent dosing is required to alleviate pain in postoperative patients. ${ }^{6}$ It is hypothesized that delivery of KT to the site of pain and inflammation may lower the incidence of hematologic and GI adverse effects that was noted in oral administration. ${ }^{20}$ Consequently, a new approach, ${ }^{21}$ which involves direct delivery of KT via adhesive films to the surgical site, was proposed for managing post-surgical pain. Moreover, the new delivery system should also provide a sustained release of this medication to assist patient compliance. Also, having $30 \mathrm{mg}$ of KT formulated into the bucco-adhesive film would have a similar therapeutic efficiency as the oral dosing regimen. To the authors' best knowledge, the influence of $30 \mathrm{mg}$ KT incorporated in adhesive film on pain management after periodontal surgery has not been established. As such, the purpose of this study is to evaluate the analgesic efficacy and tolerability of a single $30-\mathrm{mg}$ dose of KT formulated into an adhesive film for moderateto-severe postoperative pain management after periodontal surgery. Also, the authors would like to prove that their null hypothesis, that KT when used as an adhesive film is not effective in relieving pain after periodontal surgery, is not valid.

\section{MATERIALS AND METHODS}

This was a double-masked, randomized, parallel-group, placebo-controlled, single-dose, single-centered clinical trial designed based on the intention-to-treat model. The study took place from September 2009 to March 2010 at the research facility in King Saud University, Riyadh, Saudi Arabia. The study protocol was approved by the Research Center and Human Ethics Committee of the College of Pharmacy and Dentistry, King Saud University, in accordance with the Helsinki Declaration of 1972, as revised in 2000. Before enrollment, a written informed consent was obtained from the patients. Sixty-eight patients (31 male and 37 female, aged 18 to 64 years) were enrolled in this study. Patients were included based on: 1) absence or inadequate width of keratinized tissue $(<1 \mathrm{~mm})$ in the mandibular arch, from second premolar to second premolar region; 2) absence of moderate-to-severe clinical inflammation, which is defined as bleeding on probing $<25 \%$; and 3 ) absence of severe clinical attachment loss (AL) at the quadrant of interest, which is defined as clinical AL of $\geq 5 \mathrm{~mm}$. Patients with one of the following conditions were excluded from the study: 1) history of upper GI ulceration or bleeding within the past 6 months or current significant upper GI symptoms; 2) pregnant or trying to become pregnant; 3) taking analgesics or other agents that might interfere with the analgesic response (e.g., tricyclic antidepressants, narcotic analgesics, antihistamines, tranquilizers, hypnotics, sedatives, NSAIDs, or corticosteroids) within 48 hours before surgery; 4) at risk for infective endocarditis; 5) allergic to any substance of the material being tested; 6 ) initial presurgical pain intensity $<5 \mathrm{~cm}$ on the visual analog scale; and 7) smokers.

During the pretreatment period, defined as 14 days before free soft tissue graft surgery, medical and dental histories were obtained. Clinical and radiographic examinations were conducted and bleeding on probing and clinical AL were recorded. Physical examination and clinical laboratory testing for blood sugar, blood pressure, renal function, liver function, and pregnancy (for females) were performed.

Patients were randomized to two groups: test (KT film) and placebo control (film without KT). Randomization was conducted by having the patient pick a paper marked either $\mathrm{T}$ (test) or $\mathrm{C}$ (control) from a brown 


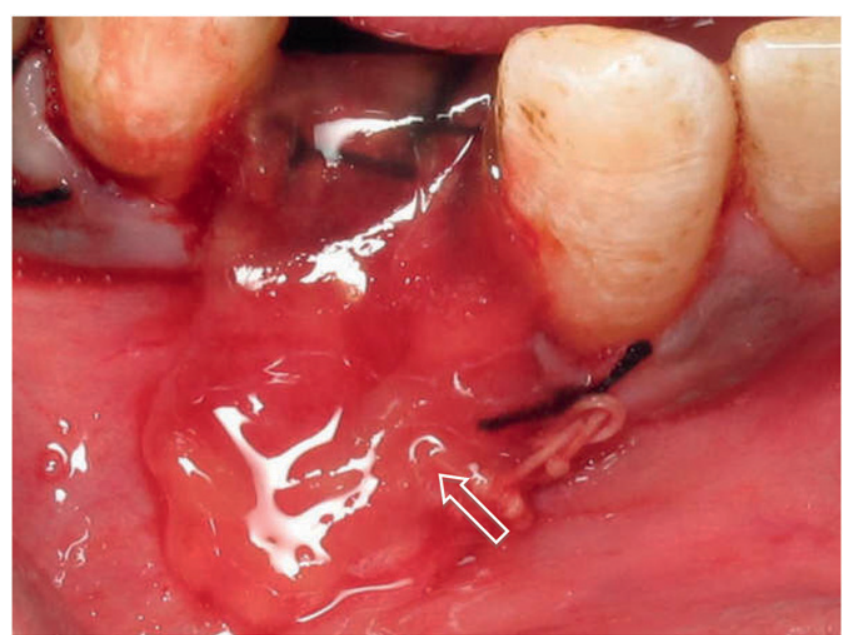

Figure I.

A ketorolac adhesive film (arrow) was applied to the site of surgery. It was easily adapted and highly adherent to the underlying tissues.

bag. The patient handed the slip of paper to research assistants (Ronilo V. Almodal and Soledad Gaspar, Department of Periodontics and Community Dentistry, College of Dentistry, King Saud University, Riyadh, Saudi Arabia), who prepared the test or placebo films accordingly. Both test and placebo films were identical in appearance. At the time of surgery, the research assistants handed the prepared film to the surgeon (KA-H) according to the randomization. The same research assistants were also responsible for monitoring the patients' response from baseline to their discharge from the research facility. The surgeon, dental and research assistants, and the patients were kept masked from the randomization process.

Free gingival grafts were recommended to increase the width of keratinized tissue in mandibular arches of the enrolled patients. At the recipient site, a partialthickness flap with vertical releasing incisions was elevated to create a connective tissue bed that was $3 \mathrm{~mm}$ apical and lateral to the defect. The free gingival grafts, with mean dimensions of 19 (length) $\times 8$ (width) $\times 1 \mathrm{~mm}$ (thickness), were harvested from the palatal tissue between the second molar and the first premolar region. Sling and periosteal suturing, using 4.0 and 5.0 chromic gut sutures, "was performed to secure the free gingival grafts firmly onto the recipient bed. For patients in the test group, the prepared KT adhesive film, ${ }^{21}$ which was trimmed to cover the free gingival grafts completely, was subsequently placed onto the graft. Patients in the control group, however, received the adhesive film without KT. The same film was placed over the donor site. Figure 1 illustrates the placement of the KT adhesive film on the recipient site.
During the treatment period, patients assessed pain intensity at baseline (immediately before the adhesive film was applied), 1, 2, 3, 4, 5, 24, and 48 hours thereafter, or immediately before taking other pain medication. All assessments were recorded in a patient logbook, which was kept in a locked facility at the research center. Pain intensity assessments were made using a standard visual analog scale where patients drew a vertical mark along a $10-\mathrm{cm}$ scale from 0 (no pain) to $10 \mathrm{~cm}$ (highest degree of pain). The research group defined mild, moderate and severe pain intensities as $>0$ to $4 \mathrm{~cm},>4$ to $7 \mathrm{~cm}$, and $>7$ to $10 \mathrm{~cm}$, respectively. For clinical safety assessments, patients were monitored closely throughout the treatment and post-treatment periods (24 and 48 hours). Vital signs, such as heart rate, blood pressure, breathing rate, and percentage of oxygen saturation, were recorded for the treated patient.

With the intention-to-treat model, patients in the control group who showed moderate-to-severe postoperative pain after the second hour post-surgery were given the adhesive film containing KT, after the placebo control film was gently removed, and pain intensity was measured again on an hourly basis. If pain intensity was $>5$ for more than two consecutive assessments after the application of KT adhesive film, the patient was excluded from the study and one pain relief tablet was given (300-mg acetaminophen and 30-mg codeine). " Patients remained at the research center and were monitored for 48 hours after receiving KT or placebo adhesive film to detect any allergic or adverse reaction. Before their discharge from the research center, physical examination and clinical laboratory testing for blood sugar level, renal and liver function, and presence of residual KT were performed.

The power calculation, using a two-sided independent $t$ test," was performed using data previously published by the authors. ${ }^{21}$ It was found that to obtain $92 \%$ power with a $P$ value set at 0.05 , a sample size of 68 patients with 34 patients in the test and control groups each was required. Efficacy analysis was performed on the intent-to-treat population, so patients who had severe pain and needed a pain relief tablet* * within 1 hour after receiving the placebo adhesive film were given the KT adhesive film. Multivariate analysis test was used to compare the results of the two groups at each time interval (95\% level of confidence). All statistical analyses were performed using a statistical package. ${ }^{\dagger \dagger}$

|| Ethicon, Johnson \& Johnson, Somerville, NJ.

Il Tylenol \#3, McNeil Consumer Healthcare, Johnson \& Johnson. \# nQuery Advisor 7.0, Statistical Solutions, Saugus, MA.

** Tylenol \#3, McNeil Consumer Healthcare, Johnson \& Johnson.

$\dagger \dagger$ SPSS version 17.0, IBM, Chicago, IL. 
Table I.

Mean and SD of Pain Intensity of Test and Control Groups at 0 (baseline), 1, 2, 3, 4, 5, 24, and 48 Hours Post-Surgery

\begin{tabular}{lcccccccc}
\hline & \multicolumn{7}{c}{ Control } & \multicolumn{1}{c}{ Test } \\
\cline { 2 - 6 } Hours Post-Surgery & Mean pain intensity \pm SD & Min. & Max. & Mean pain intensity \pm SD & Min. & Max. & $P$ Value \\
\hline 0 & $7.66 \pm 0.91$ & 6.00 & 9.50 & $7.70 \pm 0.89$ & 6.00 & 9.50 & 0.842 \\
1 & $7.82 \pm 0.68$ & 6.50 & 9.00 & $5.23 \pm 0.89$ & 4.00 & 7.00 & $0.00 I^{*}$ \\
2 & $5.63 \pm 1.38$ & 3.00 & 8.50 & $3.50 \pm 0.84$ & 2.00 & 5.00 & $0.00 I^{*}$ \\
3 & $3.41 \pm 1.31$ & 1.50 & 7.00 & $3.50 \pm 0.84$ & 2.00 & 5.00 & 0.742 \\
4 & $2.48 \pm 1.13$ & 1.00 & 6.50 & $2.04 \pm 0.81$ & 1.00 & 4.00 & 0.070 \\
5 & $1.32 \pm 0.84$ & 0.50 & 4.00 & $0.60 \pm 0.57$ & 0.00 & 2.00 & $0.001 *$ \\
24 & $0.85 \pm 0.76$ & 0.00 & 2.50 & $0.73 \pm 0.52$ & 0.00 & 2.00 & 0.462 \\
48 & $0.66 \pm 0.80$ & 0.00 & 2.50 & $0.35 \pm 0.51$ & 0.00 & 2.00 & 0.064 \\
\hline
\end{tabular}

* Significant difference observed at $P<0.05$.

\section{RESULTS}

At the end of the study, there were no reports of allergic or adverse reactions (e.g., GI erosions, ulcers and bleeding, inhibition of platelet function, and renal failure). Hence, none of the patients were discontinued from the study. Mean pain intensity for the control and test groups in the first hour postoperative was 7.82 and 5.23, respectively (Table 1 ). Intervention was made in all 34 subjects in the control group by applying the KT adhesive film after the 2-hour postoperative period. A significant reduction in pain intensity during the first 2 hours post-surgery was observed in the test group $(P<0.05)$ (Table 1 and Fig. 2$)$. After the application of KT adhesive film in the control group, pain intensity was reduced to a non-significant level compared to treatment group in the third hour post-surgery (Fig. 2). No residual KT was found in the serum analysis of all the blood samples.

\section{DISCUSSION}

This study was one of the first prospective studies in humans to evaluate the analgesic efficacy and tolerability of a single $30-\mathrm{mg}$ dose of KT formulated into an adhesive film. The results obtained in this study showed a significant reduction of pain intensity means by KT adhesive film compared to a placebo (adhesive film only). The results of our study were in agreement with Mulshine et al. ${ }^{20}$ which reported that the prepared KT adhesive films were well tolerated in all patients with no complaints of GI side effects or any allergic and adverse reaction on the oral tissue.

KT is an NSAID that blocks the COX pathway and is responsible for prostaglandin production. ${ }^{6}$ It is formulated in an adhesive film to overcome the COX- 1-mediated adverse reactions, such as Gl erosions, bleeding, and ulcerations, hence increasing patient compliance. The film was formulated using aqueous solvents by means of two bioadhesive polymers (hydroxylpropyl methylcellulose and polyacrylic acid ${ }^{\ddagger}$ ) and was subjected to physical and mechanical testing for its behavior in swelling; in vitro bioadhesion; and in vitro, in situ, and in vivo release. ${ }^{21}$ Anti-inflammatory efficacy and analgesic activity of the prepared adhesive film were investigated using the hind-paw edema method of rats and the hot plate method. 22 The analgesic efficacy and tolerability of single 30mg dose of KT adhesive film was clinically evaluated in a pilot study, ${ }^{21}$ and it was found that the film did not cause any adverse reaction or GI side effects.

According to previous work by our group, ${ }^{21}$ the buccoadhesive ketorolac film had good adhesion, near neutral $\mathrm{pH}$, and reasonable ketorolac release. Its detachment force was $22 \pm 2.3$ dyne $/ \mathrm{cm}^{2}$ with a pH of 6.0. In addition, its concentration in the oral cavity was maintained $>4 \mathrm{mg} / \mathrm{mL}$ for at least 6 hours, where $85 \%$ to $90 \%$ of ketorolac was released. Because maximum drug concentration was $130.6 \pm 22.2 \mathrm{mg} /$ $\mathrm{mL}$ and time of occurrence for peak drug concentration was 1.5 hours, 2 hours was chosen as the time point when rescue therapy was rendered. ${ }^{21}$ As such, KT adhesive film applied to the surgical site offers several advantages compared to its conventional routes of administration (e.g., orally or intramuscularly). These advantages include minimal drug interaction, less GI upset, less bleeding concerns, high patient compliance, and effectiveness in controlling

† Carbopol 934 NF Polymer, GM Chemical, Yangzhou, China. 
A

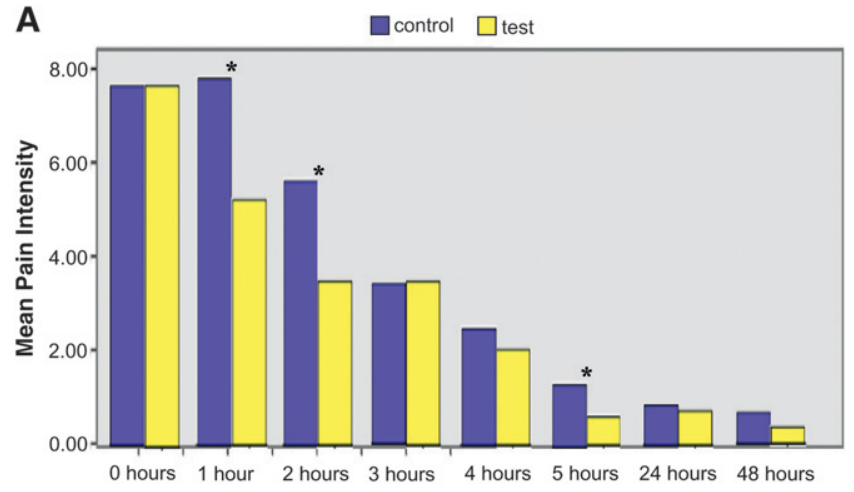

B



Figure 2.

A) Bar chart demonstrating the pain intensity mean for both test and control groups at 0 (baseline), I, 2, 3, 4, 5, 24, and 48 hours post-surgery. The test and control groups are represented by the yellow and blue bars, respectively. * Significant difference was observed between the test and control groups $(P<0.05)$. B) Box plot diagram demonstrating the range of pain intensity outcome values for both test and control groups at 0 (baseline), I, 2, 3, 4, 5, 24, and 48 hours post-surgery. The test and control groups are represented by the yellow and blue bars, respectively. The horizontal line within each box represents the median pain intensity value. The upper and lower ends of every box corresponded to the 25th and 75 th percentile of each group at the various time points. * Significant difference was observed between the test and control groups $(\mathrm{P}<0.05)$.

moderate-to-severe post-surgery pain. It was interesting to observe from this study that all patients in the control group experienced moderate-to-severe pain, and intervention using the KT adhesive film was required at 2 hours post-surgery. In addition, after the film was applied, discomfort was significantly reduced to a comparable level as in the test group. This clearly demonstrates the effectiveness of the KT film in controlling pain after periodontal surgery.

\section{CONCLUSION}

The KT adhesive films, in a single dose of $30 \mathrm{mg}$, were an effective way of controlling post-surgical pain with no observable GI side effects, such as GI erosions, ulcers, and bleeding.

\section{ACKNOWLEDGMENTS}

This study was supported by the Eng. A.B. Growth Factors and Bone Regeneration Center, Division of Periodontics, College of Dentistry, King Saud University, Riyadh, Saudi Arabia. The authors report no conflicts of interest related to this study.

\section{REFERENCES}

1. Wessel JR, Tatakis DN. Patient outcomes following subepithelial connective tissue graft and free gingival graft procedures. J Periodontol 2008;79:425-430.

2. Griffin TJ, Cheung WS, Zavras AI, Damoulis PD. Postoperative complications following gingival augmentation procedures. J Periodontol 2006;77:2070-2079.

3. Pihlstrom BL, Hargreaves KM, Bouwsma OJ, Myers WR, Goodale MB, Doyle MJ. Pain after periodontal scaling and root planing. J Am Dent Assoc 1999;130:801-807.

4. Fitzgerald DJ, Maree A. Aspirin and clopidogrel resistance. Hematology Am Soc Hematol Educ Program 2007:114-120.

5. Mroszczak EJ, Jung D, Yee J, Bynum L, Sevelius H, Massey I. Ketorolac tromethamine pharmacokinetics and metabolism after intravenous, intramuscular, and oral administration in humans and animals. Pharmacotherapy 1990;10:33S-39S.

6. Buckley MM, Brogden RN. Ketorolac. A review of its pharmacodynamic and pharmacokinetic properties, and therapeutic potential. Drugs 1990;39:86-109.

7. Alanazi FA, Rahman AAA, Mahrous GM, Alsarra IA. Formulation and physicochemical characterization of buccaadhesive films containing ketorolac. J Drug Del Sci Tech 2007; 17:183-192.

8. Walton GM, Rood JP, Snowdon AT, Rickwood D. Ketorolac and diclofenac for postoperative pain relief following oral surgery. Br J Oral Maxillofac Surg 1993; 31:158-160.

9. Pilotto A, Franceschi M, Leandro G, et al. The risk of upper gastrointestinal bleeding in elderly users of aspirin and other non-steroidal anti-inflammatory drugs: The role of gastroprotective drugs. Aging Clin Exp Res 2003;15:494-499.

10. Olmedo MV, Gálvez R, Vallecillo M. Double-blind parallel comparison of multiple doses of ketorolac, ketoprofen and placebo administered orally to patients with postoperative dental pain. Pain 2001;90:135-141.

11. Roy SD, Manoukian E. Transdermal delivery of ketorolac tromethamine: Permeation enhancement, device design, and pharmacokinetics in healthy humans. J Pharm Sci 1995;84:1190-1196.

12. Amrish C, Kumar SP. Transdermal delivery of ketorolac. Yakugaku Zasshi 2009;129:373-379.

13. Cho YA, Gwak HS. Transdermal delivery of ketorolac tromethamine: Effects of vehicles and penetration enhancers. Drug Deu Ind Pharm 2004;30:557-564.

14. Tiwari SB, Pai RM, Udupa N. Influence of ultrasound on the percutaneous absorption of ketorolac tromethamine in vitro across rat skin. Drug Deliv 2004;11:47-51.

15. Santus G, Rivolta R, Bottoni G, Testa B, Canali S, Peano S. Nasal formulations of ketorolac tromethamine: Technological evaluation - Bioavailability and tolerability in rabbits. Farmaco 1993;48:1709-1723.

16. Chelladurai S, Mishra M, Mishra B. Design and evaluation of bioadhesive in-situ nasal gel of ketorolac tromethamine. Chem Pharm Bull (Tokyo) 2008;56: 1596-1599. 
17. Cordero JA, Camacho M, Obach R, Domenech J, Vila L. In vitro based index of topical anti-inflammatory activity to compare a series of NSAIDs. Eur $J$ Pharm Biopharm 2001;51:135-142.

18. Perry HD, Donnenfeld ED. An update on the use of ophthalmic ketorolac tromethamine 0.4\%. Expert Opin Pharmacother 2006;7:99-107.

19. Jeffcoat MK, Reddy MS, Haigh S, et al. A comparison of topical ketorolac, systemic flurbiprofen, and placebo for the inhibition of bone loss in adult periodontitis. $J$ Periodontol 1995;66:329-338.

20. Mulshine JL, Atkinson JC, Greer RO, et al. Randomized, double-blind, placebo-controlled phase Ilb trial of the cyclooxygenase inhibitor ketorolac as an oral rinse in oropharyngeal leukoplakia. Clin Cancer Res 2004; 10:1565-1573.
21. Alsarra IA, Alanazi FK, Mahrous GM, Abdel Rahman AA, Al Hezaimi KA. Clinical evaluation of novel buccoadhesive film containing ketorolac in dental and post-oral surgery pain management. Pharmazie 2007; 62:773-778

22. Peh KK, Wong CF. Polymeric films as vehicle for buccal delivery: Swelling, mechanical, and bioadhesive properties. J Pharm Pharm Sci 1999;2:53-61.

Correspondence: Dr. Khalid Al-Hezaimi, Eng. A.B. Growth Factors and Bone Regeneration, King Saud University, P.O. Box 103851, Riyadh 11616, Saudi Arabia. Fax: 966 1-4677601; e-mail: alhezaim@usc.edu.

Submitted September 8, 2010; accepted for publication November 13, 2010. 